\title{
MENTES BRILLANTES (2018): UNA ODISEA PARA CONSEGUIR UNA PLAZA EN MEDICINA
}

\section{Brilliant minds (2018): An odyssey to get enrolled in medicine}

Emilio PINTOR HOLGUÍN; Nieves MARTínEZ ALSINA; María Josefa GUTIÉRREZ CISNEROS; Benjamín HERREROS RUIZ-VALDEPEÑAS

Universidad Europea de Madrid. Facultad de Ciencias Biomédicas. Villaviciosa de Odon. Madrid (España).

Autor para correspondencia: Emilio Pintor Holguín.

Correo electrónico: emilio.pintor@universidadeuropea.es

Recibido el 14 de abril de 2020

Aceptado el 21 de abril de 2020

\section{Resumen}

Antoine y Benjamin son dos jóvenes franceses que, tras haber terminado sus estudios de bachillerato en ciencias, coindicen en el curso preparatorio para elegir su futura carrera universitaria. Solo aquellos que consigan las mejores notas y por tanto los mejores puestos al final del curso podrán elegir medicina. Mientras que para Antoine; que no tiene una mente especialmente brillante, pero si una capacidad de sufrimiento y de estudio ilimitado, el conseguir una plaza para estudiar medicina es lo más importante en su vida, para Benjamin; cuyo padre es cirujano digestivo, y le resulta relativamente fácil sacar buenas notas, no tiene clara su vocación. La película plantea una crítica sobre cuáles son los parámetros que determinan quienes deben ser aceptados para estudiar medicina así como la creciente competitividad entre los posibles candidatos, donde a la hora de la verdad, tus amigos pasan a ser competidores y por tanto enemigos.

Palabras clave: medicina; estudiantes de medicina; exámenes de ingreso; competitividad.

\section{Summary}

Antoine and Benjamin are two french teenagers, after having finished their high school studies in science, who met in the preparatory course for their future university career. Only those who get the best grades and therefore the best positions at the end of the course will be able to choose medicine. While for Antoine; that he does not have an especially brilliant mind, but if an unlimited capacity for suffering and study, getting a place to study medicine is the most important thing in his 
MENTES BRILLANTES (2018): UNA ODISEA PARA CONSEGUIR UNA PLAZA EN MEDICINA

EMILIO PINTOR HOLGUÍN; NIEVES MARTÍNEZ ALSINA; MARÍA JOSEFA GUTIÉRREZ CISNEROS; BENJAMÍN HERREROS RUIZ-VALDEPEÑAS

life, for Benjamin; whose father is a digestive surgeon, and it is relatively easy for him to get good grades, his vocation is unclear. The film raises a criticism about what are the parameters that determine who should be accepted to study medicine as well as the increasing competitiveness among potential candidates, where at the end, your friends become competitors and therefore enemies.

Keywords: medicine; medical students; admission exams; competitiveness.

Ficha técnica

Título: Mentes brillantes.

Titulo original Première année.

País: Francia.

Año: 2018.

Director: Thomas Lilt.

Musica: Alexandre Lier, Sylvain Ohrel, Nicolas Weil.

Fotografía: Nicolas Gaurin.

Montaje: Christel Dewynter.

Guion: Thomas Lilti

Interpretes: Vincent Lacoste, William Lebghil, Michel Lerousseau, Darina El Joundi, Benoît Di Marco, Graziella Delerm, Guillaume Clérice, Alexandre Blazy, Noémi Silvania, ...

Género: drama

Idioma original: francés.

Color: color.

Duración: 92 minutos.

Sinopsis: Antoine decide prepararse para las pruebas de acceso a Medicina... por tercera vez. En cambio, para Benjamin es su primer intento y pronto se dará cuenta de que esto no es pan comido. En un mundo tan competitivo y caracterizado por noches de estudio intenso en lugar de fiestas universitarias, los dos ami- gos tendrán que encontrar un término medio entre el sacrificio del presente y la esperanza de un brillante futuro.

Productoras: 31 Juin Films, Les Films du Parc, France 2 Cinéma, Le Pacte, Les Films de Benjamin, Canal+ / Ciné+, France Télévisions, ...

\section{Enlaces:}

https://www.imdb.com/title/tt6690004/

https://www.filmaffinity.com/es/film249608. html

$\underline{\text { Trailer }}$

\section{Introducción}

Thomas Lilti; el director de la película, antes de ser cineasta realizó su formación en el grado de Medicina y después ejerció durante un tiempo de médico de familia. Eso ha influido de forma fundamental en 3 de sus películas: Hipócrates (2014), Médico de la campiña (2016) y esta última estrenada en 2018 que es Mentes brillantes. En cada una de ellas trata con realismo; no exento de imágenes y situaciones de humor negro, ironía o surrealismo, problemas o debates existentes en la sanidad francesa.

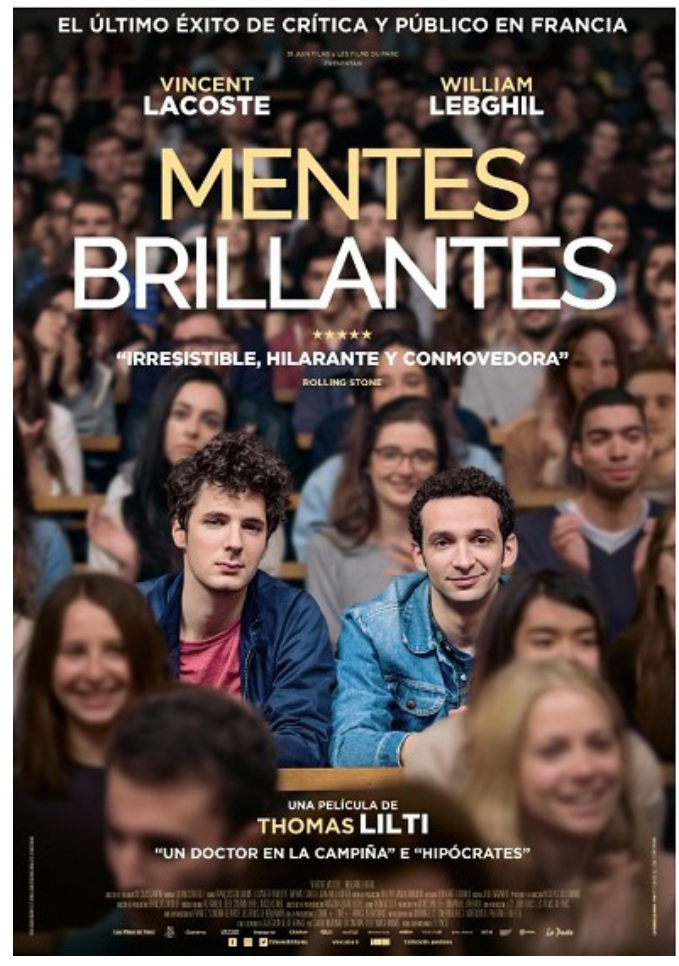

Cartel español. 
En Hipócrates; ya comentada por nuestro grupo en esta revista ${ }^{1}$, a través de los ojos de 2 residentes de un hospital público se reflexiona sobre el encarnizamiento terapéutico, las negligencias médicas y los recortes en la sanidad pública. En Médico en la campiña ${ }^{2}$, plantea el antagonismo entre la medicina despersonalizada hospitalaria y la medicina rural donde el médico trabaja 24 horas al día y acompaña al enfermo no solo en los problemas médicos.

Por último, en la película que hoy comentamos; Mentes brillantes, el debate que plantea es la forma de elección de los candidatos que luego poblaran las escuelas de Medicina de Francia. En esta película se describe el día a día de los 2 personajes fundamentales; Antoine (Vincent Lacoste) y Benjamin (William Lebghil), a lo largo de su curso preparatorio para optar a una plaza para estudiar medicina.

Antoine y Benjamin: dos mundos y personalidades antagónicas

El primer protagonista es Antoine, un chico para el que estudiar medicina es el sentido fundamental de su vida y que intentará hacer todo lo posible para conseguir una plaza. Este es el tercer año que va a presentarse a los exámenes del curso preparatorio ya que en los 2 años anteriores que quedó a las puertas de elegir medicina y renunció a poder hacer otra disciplina de ciencias de la salud (odontología, enfermería, farmacia, ....). Es un joven con una familia normal que para conseguir su sueño está dispuesto a estudiar prácticamente 24 horas al día sin hacer ninguna otra actividad. Tiene vocación de poder ser médico, pero a pesar de su sacrificio constante en los exámenes no saca los resultados deseados.

El segundo personaje se llama Benjamin. Este adolescente; hijo de un cirujano digestivo, tiene una mente brillante que le permite obtener buenos resultados en los estudios. No tiene claro su futuro profesional y carece de vocación de médico. Quizás piensa hacer medicina para satisfacer los deseos de su padre, pero a lo largo de la película en múltiples momentos se refleja el desprecio de su padre a pesar de los buenos resultados porque no ve en él vocación ni madera de médico. Para él, además de estudiar existen otras cosas; la comida, las relaciones sociales, los videojuegos, etc.

En una escena de la película que parece una alegoría al cuadro de Rembrant: "Lección de anatomía del Dr. Nicolaes Tulp», se reflejan estas 2 personalidades distintas. Antoine lleva a Benjamin a ver a través de la puerta entreabierta una disección de un cadáver humano (Fotos 1 y 2). En esta escena, mientras Antoine mira embobado la disección desde lejos, anhelando el poder estudiar medicina y verse algún día entre esos alumnos disecando un cadáver, Benjamín muestra una total indiferencia y el único comentario que hace es su repulsión por el mal olor (formol).

Exámenes de admisión a Medicina: ¿Cuál es el sistema «menos malo»?

Al existir limitación en el número de alumnos que quieren estudiar medicina cada año en las facultades públicas y privadas debe haber algún sistema de selección entre los candidatos. EI problema es definir cuál es sistema para elegir a los que en el futuro serán los mejores médicos.

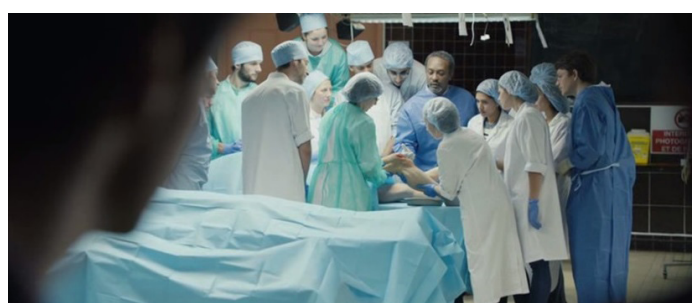

Foto 1. Antoine extasiado mirando una disección humana. 


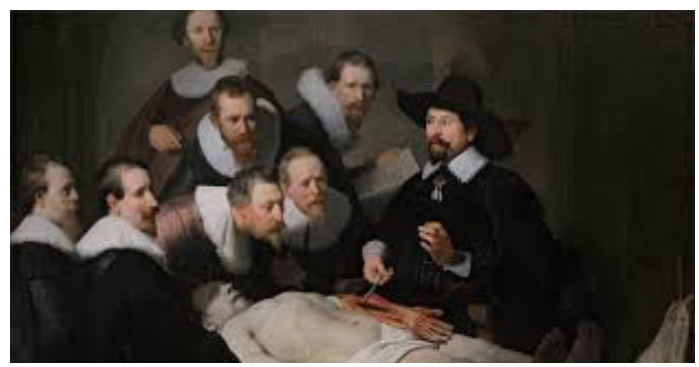

Foto 2. Cuadro de Rembrant «Lección de anatomía».

En el sistema francés, según se narra en la película, hay un curso preparatorio en el que se estudian diferentes disciplinas de ciencias (anatomía, física, química, fisiología, ...) y al final del curso se hace un examen tipo test de opción múltiple en 3 horas. Al haber más de 2000 candidatos y solo 234 plazas de medicina, solo los mejores podrán elegir medicina. El resto tendrán que elegir otras disciplinas sanitarias o renunciar y presentarse al año siguiente.

Al principio de la película aparece el decano «amenazando con las estadísticas» en sus primeras palabras:

"... os habrán explicado vuestros orientadores que éste es un curso difícil ... la carrera de medicina os someterá a mucha presión ... las posibilidades de superar el primer curso en aquellos que no vengan con sobresaliente del bachillerato son del $2 \%$...»

Durante varios momentos de la película se discute sobre si lo importante es aprender o aprender a aprobar los test. Así, ésta es una de las conversaciones en que se trata este tema:

Benjamin se encuentra con un amigo de su hermano, al que le cuenta que está haciendo el curso preparatorio para hacer medicina:

Amigo: "¿Sabes que es lo que diferencia el alumno de medicina del de preparatorio? "

Benjamin: No.

Amigo: "Si les pides que se aprendan la guía telefónica, el de preparatorio te preguntará para qué y el de medicina ... para cuándo».

Amigo: "Al final solo se os juzga por la capacidad de digerir montañas de información que después no servirán de nada ... como calcular la desviación de las ondas sobre un estanque».

"iAh! Un consejo, haz exámenes anteriores, "aprende, no pierdas el tiempo intentando comprender", ese es el secreto».

En nuestro país, la elección de plaza en la universidad se establece en función de las notas del bachillerato y la prueba de selectividad. Este no es un examen test como el que se representa en la película.

En España, una situación parecida a la que presenta la película; un examen tipo test con muchos aspirantes, sería el examen MIR en el que los graduados en medicina, se jugarían su futuro como especialistas en un examen. La preparación del examen MIR se ha establecido en nuestros días como un examen en que lo importante es; como dicen en la película, hacer muchos test para poder sacar una nota mejor. De hecho, hay academias de preparación para el MIR en las que se estimula la realización de muchos test, cuantos más, mejor. En los últimos años para evitar esto, al menos en cierta medida, es introducir preguntas que estén basadas en el racionamiento clínico no exclusivamente en la memoria ${ }^{3,4}$.

\section{Preparación de un examen "vital» para el futuro profesional}

En las últimas semanas o incluso meses antes de este tipo de exámenes (en Francia de ingreso en medicina o en España de selección de especialidad médica (MIR) o en otro tipo de oposiciones), la preparación se convierte en un estudio 24 horas al día sin ningún tiempo para ninguna otra cosa. Esto implica un árido esfuerzo de estudio, descontextualizado de la vida académica y del contacto clínico. Se trata de mantenerse durante meses sujeto a una disciplina solitaria a fin de 
poder competir con los colegas en un examen teórico. El buen temple emocional y la capacidad organizativa son los auténticos protagonistas del desafío (Fotos 3 y 4 ).

Sin embargo, no todos los alumnos pueden soportar mentalmente semejantes desafío ${ }^{5}$. Se ha publicado que un porcentaje importante de sujetos sometidos a situaciones tan estresantes desarrollaran trastornos de depresión y ansiedad, así como otras enfermedades mentales como trastornos de la conducta alimentaria o incluso cuadro psicóticos agudos. De hecho, en algunas universidades como la de Barcelona han realizado talleres psico-educativos voluntarios dirigidos a los alumnos de 60 curso que afrontaban la preparación del examen MIR con los que se pretenden un doble objetivo: fomentar una organización efectiva del trabajo de preparación del examen y promover el aprendizaje de herramientas que permitieran manejar el estrés y la ansiedad que implica la preparación del MIR ${ }^{7,8}$.

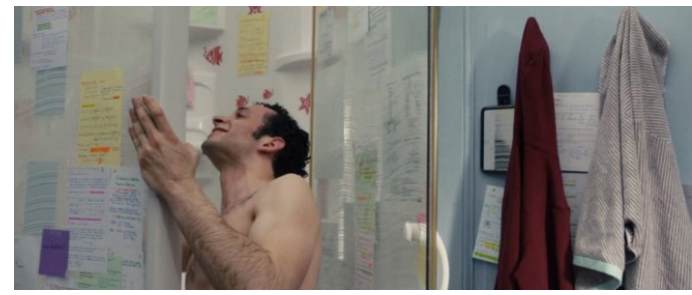

Foto 3. Benjamin saliendo de la ducha «empapelada» con apuntes.

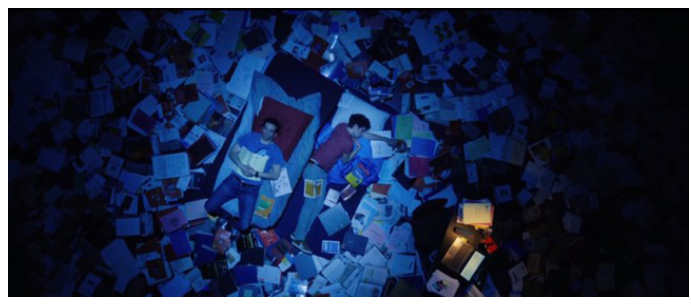

Foto 4. Habitación de Antoine en la días previos al examen.

Este hecho está reflejado en la película en el personaje de Antoine. Los malos resultados a pesar del esfuerzo titánico generan ansiedad y angustia que determinan en Antoine un cambio de personalidad. El carácter se agria en especial con sus padres y compañeros. Se aísla y en un momento determinado presenta en clase un cuadro alteración mental transitoria (probablemente un brote psicótico) que requiere de ingreso hospitalario en unidad de psiquiatría (Foto 5).

Espacio para la crítica utilizando un humor surrealista

Lilti, como ya pudimos ver en Hipócrates ${ }^{1}$, introduce en sus películas algunos momentos hilarantes. Uno es en relación con la compra de un libro. Antoine va a una librería a comprar un libro de química (Foto 6) y resulta el libro está publicado en 6 editoriales diferentes y ésta es la conversación con el librero:

"¿el más completo? ... y el más caro ¿es?... pero el Masson es el más corto... ¿y cuál se vende más?... ¿y el más reciente?»

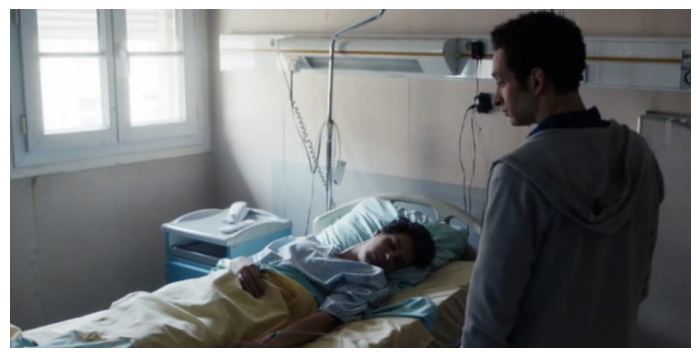

Foto 5. Benjamin acude a visitar a Antoine en su ingreso hospitalario por cuadro psicótico agudo.

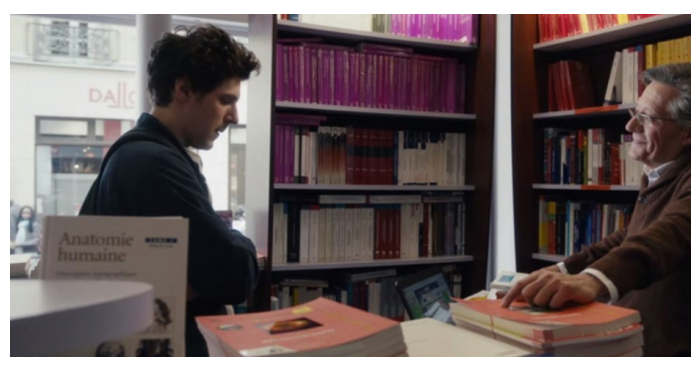

Foto 6. Antoine en la librería comprando un libro de química. 
Otro tema que trata con humor es la compra de las fotocopias de los apuntes (Foto 7). Antoine y Benjamín acuden al servicio de reprografía de la universidad a adquirir las fotocopias de los apuntes y éste es el diálogo entre la encargada y ellos:

Antoine: "¿Oiga son las mismas que el año pasado? Las fotocopias, porque si son las mismas del año pasado, ya las tengo».

Señora: «Es posible».

Antoine: "¿qué es posible?»

Señora: «Es posible que sean las mismas».

Antoine: "Pues no las voy a comprar».

Señora: «... o que haya habido cambios. No lo sé».

Benjamin: «¿Quiere decir que no sabe si ha habido cambios?»

Señora: «Eso es».

Antoine: "Entonces, cंlas compramos?»

Señora: "Como queráis».

Otra alumna de la cola: "Ya que estáis aquí llevároslas, venga».

Antoine: «Un segundo. Y ¿puede que haya habido pocos cambios?"

Señora: "... o ninguno ... o muchos, no nos lo dicen".

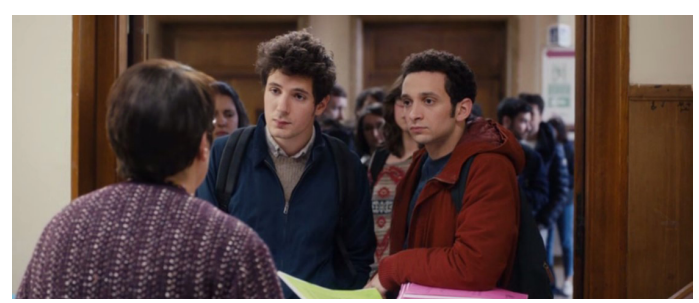

Foto 7. Antoine y Benjamin con la encargada de reprografía.

Por último, justo antes de las vacaciones de Navidad en la última clase, un grupo de alumnos disfrazados irrumpen en la clase y hacen que le profesor y el resto de los alumnos canten una canción de contenido satírico sexual (Foto 8).

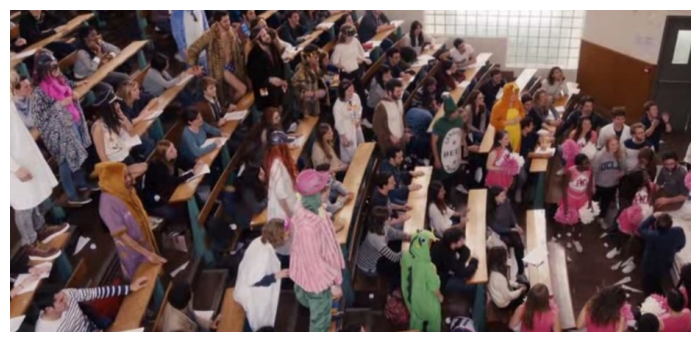

Foto 8. Aula del curso preparatorio. Alumnos disfrazados hacen cantar al profesor.

\section{Referencias}

1. Pintor-Holguin E, Herreros-Ruiz B, Gargantilla-Madera P, Vivas-Rojo E. Hipócrates (2014). La realidad de un residente en un hospital público. Rev Med Cine. [Internet] 2017;13(2014):171-6.

2. García-Torres D. Original Intercambios sobre la filmografía de Thomas Lilti, su incidencia en la formación de médicos cubanos. Rev Med Cine.[Internet] 2019;15(2):89-93.

3. Aranda M. Examen al examen MIR . Med Clin (Barc) 2016;146(10):443-5.

4. Casado Vicente V. El examen MIR y las plazas de médico de familia. Aten primaria. 2006;38(10):535-6.

5. Sender $R$, Valles $A$, Puig $O$, Salamero $M$, Valdés $M$. ¿Qué hay detrás del miedo a los exámenes? Educ Med. 2004;7(1):36-41.

6. González-Cabrera J, Fernández-Prada M, Iribar-Ibabe C, Peinado JM. Acute and chronic stress increase salivary cortisol: a study in the real-life setting of a national examination undertaken by medical graduates. Stress. 2014;17(2):149-56.

7. Diaz L, Vallés A, Sender R, M. S. Presentación y evaluación de un curso para aprender a controlar la ansiedad durante la preparación del examen MIR. Educ Med. 2001;4(3):119.

8. Sender R, Salamero M. Programa de Atención Psicológica para los alumnos de la Facultad de Medicina de la Universidad de Barcelona. Educ Med. 2007;10(4):252-7. 


\begin{tabular}{|c|c|}
\hline & $\begin{array}{l}\text { Emilio Pintor Holguín. Licenciado y doctor en Medicina. Especialista en Medi- } \\
\text { cina Interna. Catedrático en Medicina Interna y profesor de fisiopatología en } \\
\text { la Universidad Europea de Madrid. Ha publicado diferentes estudios sobre } \\
\text { la influencia y la utilidad del cine y las series médicas en la formación de } \\
\text { estudiantes de ciencias de la salud. }\end{array}$ \\
\hline & $\begin{array}{l}\text { Nieves Martinez Alsina. Licenciada en Medicina y Cirugía. Especialista en } \\
\text { Medicina de Familia y Comunitaria. Profesora adjunta en Medicina en la } \\
\text { Universidad Europea de Madrid. }\end{array}$ \\
\hline & $\begin{array}{l}\text { María José Gutiérrez Cisneros. Licenciada y doctora en Farmacia. Especialista } \\
\text { en Microbiología y Parasitología Clínica. Profesora adjunta de Parasitología } \\
\text { en la Universidad Europea de Madrid. }\end{array}$ \\
\hline$=\left(\begin{array}{ll}1 & 1\end{array}\right)$ & $\begin{array}{l}\text { Benjamín Herreros Ruiz-Valdepeñas. Licenciado y doctor en Medicina y } \\
\text { cirugía. Especialista en Medicina Interna. Director del Instituto de Ética } \\
\text { Clínico Francisco Vallés-UE. Profesor de humanidades médicas y bioética, } \\
\text { coordina la asignatura Cine y Medicina en la Universidad Complutense de } \\
\text { Madrid. Autor de los libros «El médico en el cine» y «El enfermo en el cine». }\end{array}$ \\
\hline
\end{tabular}

